\title{
U-Shape Kissing Chimney Thoracic Endovascular Aneurysm Repair for a Traumatic Arch Rupture in a Polytraumatized Patient
}

\author{
Jeroen M. H. Hendriks, MD, PhD ${ }^{1 *}$, Tim Brits, $M D^{1}$, Thijs Van der Zijden, $\mathrm{MD}^{2}$, \\ Koen Monsieurs, MD, PhD ${ }^{3}$, Dina de Bock, MD ${ }^{4}$, Rudi De Paep, MD ${ }^{5}$ \\ ${ }^{1}$ Department of Thoracic \& Vascular Surgery, Antwerp University Hospital, Antwerp, Belgium \\ ${ }^{2}$ Department of Interventional Radiology, Antwerp University Hospital, Antwerp, Belgium \\ ${ }^{3}$ Department of Emergency Medicine, Antwerp University Hospital, Antwerp, Belgium \\ ${ }^{4}$ Department of Cardiac Surgery, Antwerp University Hospital, Antwerp, Belgium \\ ${ }^{5}$ Intensive Care Department, Antwerp University Hospital, Antwerp, Belgium
}

\begin{abstract}
An 18-year-old male patient was admitted to our hospital because of a high impact trauma. A computed tomography scan showed massive mediastinal bleeding due to a posteriorly located rupture of the aortic arch with formation of a pseudoaneurysm. Although urgent repair was indicated, open cardiac surgery was not feasible, as this would involve full heparinization in a patient with subarachnoid bleeding. The chosen solution was to perform a percutaneous thoracic endovascular aneurysm repair (TEVAR) and a kissing chimney procedure using a U-shape configuration.

Copyright $\odot 2015$ Science International Corp.
\end{abstract}

\section{Key Words}

Aortic Arch Rupture • Percutaneous TEVAR • Chimney

\section{Introduction}

An 18-year-old male patient was admitted to our hospital because of a high impact trauma in which he fell off his motorcycle at high speed. Clinically, an open femur shaft fracture was visualized. Due to a Glasgow coma scale of $3 / 15$ with severe signs of pain and hemodynamic instability, the patient was intubated on site.
The trauma protocol, including total body computed tomography $(\mathrm{CT})$, revealed the following lesions: displaced femoral shaft, tibial fracture of the left lower extremity, diffuse axonal injury, and bifrontal subarachnoid hemorrhage. Besides these lesions, massive mediastinal bleeding was seen. This was due

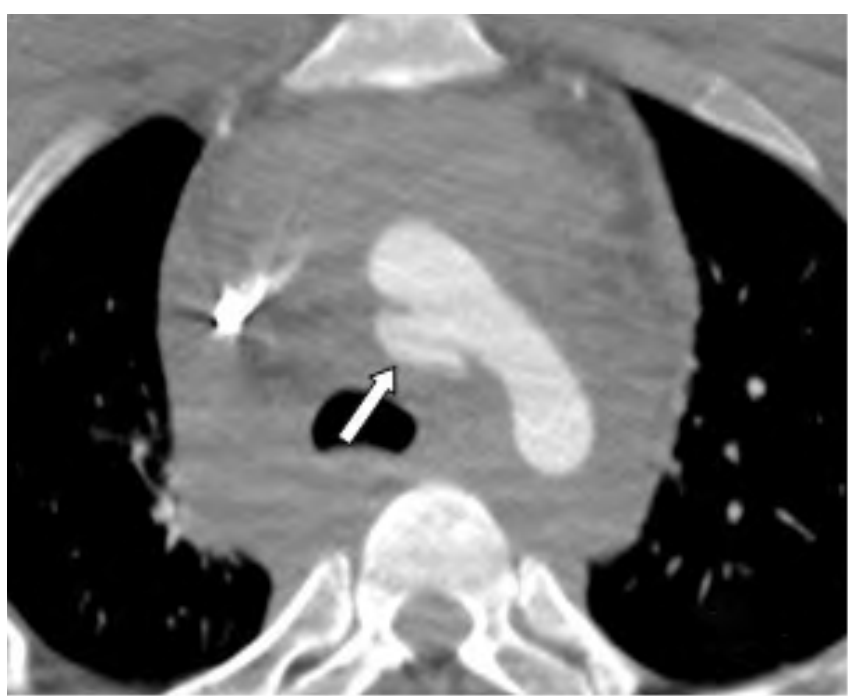

Figure 1. Mediastinal bleeding caused by a rupture of the aortic arch. White arrow: Posterior wall pseudoaneurysm.

\section{* Corresponding Author:}

Jeroen M. H. Hendriks, MD, PhD Department of Thoracic and Vascular Surgery Antwerp University Hospital

Wilrijkstraat 10, 2650 Edegem, Belgium

Tel. +32 38213785 , Fax +32 38214396, E-Mail: jeroen.hendriks@uza.be 


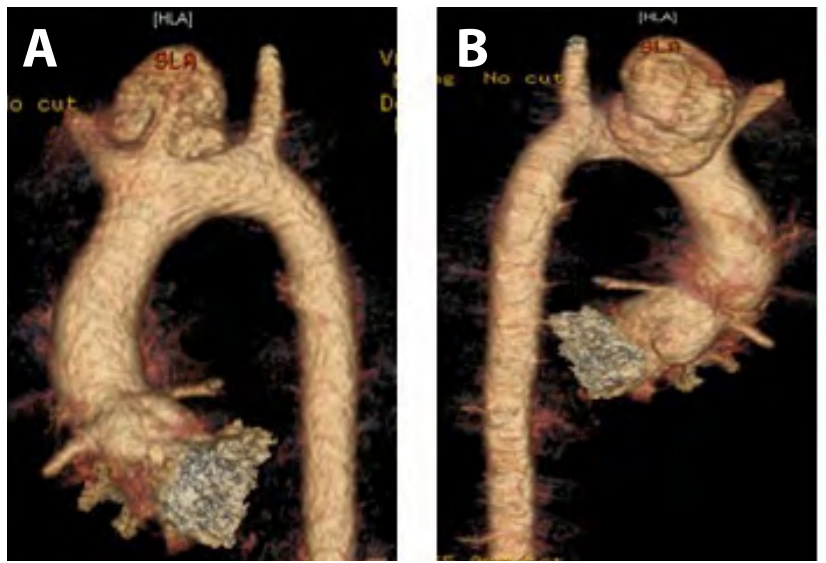

Figure 2. (T-reconstruction, note the bovine arch and rupture in close proximity to the brachiocephalic trunk. A) anterior view. B) posterior view.

to a posteriorly located rupture of the aortic arch with formation of a pseudoaneurysm (Figure 1).

Due to hemodynamic instability, urgent repair was indicated. Open cardiac surgery, however, was contraindicated, as this would involve circulatory arrest and full heparinization. Anticoagulation therapy was especially hazardous because of the subarachnoid bleeding, unknown cerebral damage, and substantial orthopedic trauma. Therefore, an endovascular approach was favored.

A more detailed examination of the $\mathrm{CT}$ scan showed a bovine arch with common origin of the brachiocephalic trunk and the left common carotid artery (LCCA), complicating the procedure (Figure 2). Debranching of the LCCA was not possible because the rupture was too close to the origin of the brachio cephalic trunk, thus inducing an unacceptable proximal landing zone (Figure 2).

The chosen solution was to perform a kissing chimney procedure and percutaneous TEVAR.

At first, an endoprosthesis (Medtronic Valiant Captiva) was delivered percutaneously via the right common femoral artery after preclosure with two ProGlides (Abbott Perclose ProGlide Suture-Mediated Closure System). Next, we performed chimney stenting of the LCCA (Advanta V12 - 6x59) through access in the left internal carotid artery. After this procedure, substantial leakage was still visible, and, when switching the sheath to provide access to the LCCA, the V12 stent luxated, possibly due to undersizing.

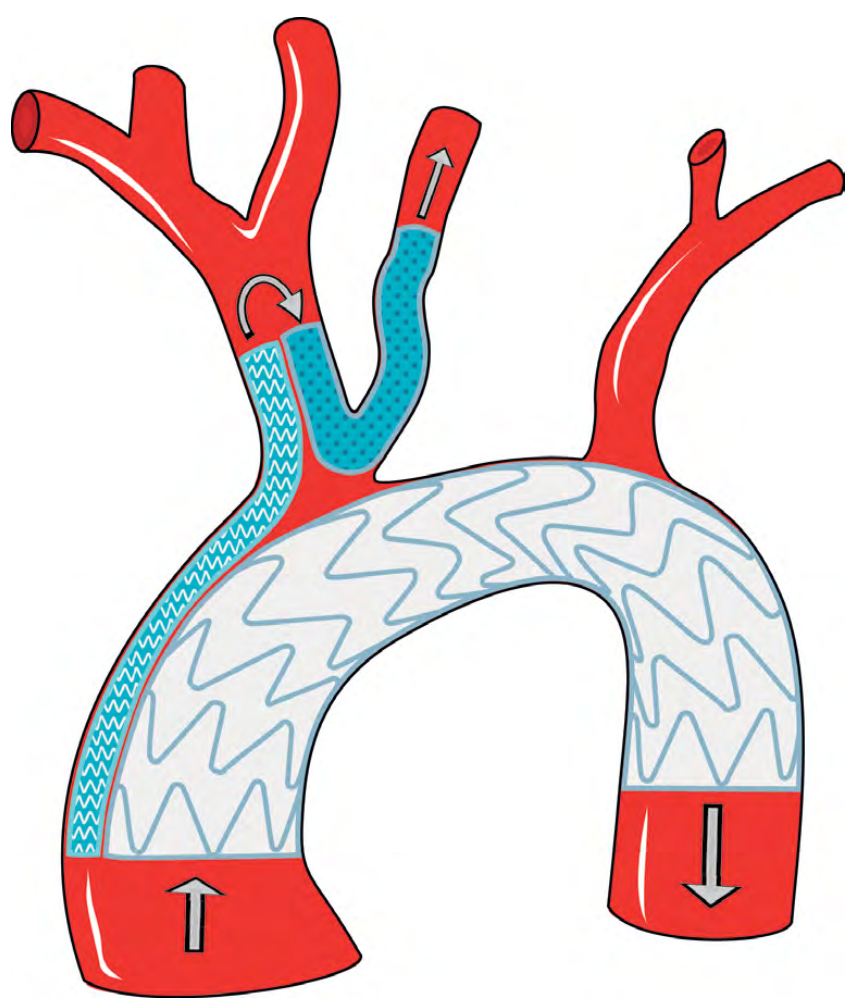

Figure 3. Schematic diagram of the construction used in this patient: Large waved area: 2 subsequent endoprostheses (Valiant Captivia, Medtronic, Minneapolis, Minnesota, USA) overriding the brachiocephalic trunk. Small waved area: 2 Advanta V12 stents from the ascending aorta, entering the brachiocephalic trunk. Dotted area: U-shaped Viabahn stent allowing blood to enter the LCCA from the brachiocephalic trunk. Arrows: blood

Therefore a more complex option was chosen: This began with an elongation of the endoprosthesis with a second Valiant Captivia placed more distally and overriding the brachiocephalic trunk, which meant placing kissing chimney stents. At the most proximal part of this "kissing chimney" configuration were two Advanta V12 10x38mm and 10x59mm stents (Maquet Cardiovascular, Wayne, New Jersey, USA) introduced via the right brachial and subclavian arteries. This allowed for blood from the ascending aorta to enter the trunk and proceed through the right subclavian artery and the right common carotid artery. The second portion of the chimney allowed blood to enter the LCCA from the trunk in a retrograde manner. This part consisted of a $7 \times 100 \mathrm{~mm}$ Viabahn stent (Gore Medical, Flagstaff, Arizona, USA) introduced after puncture of the LCCA and wiring of this vessel back and upwards, 


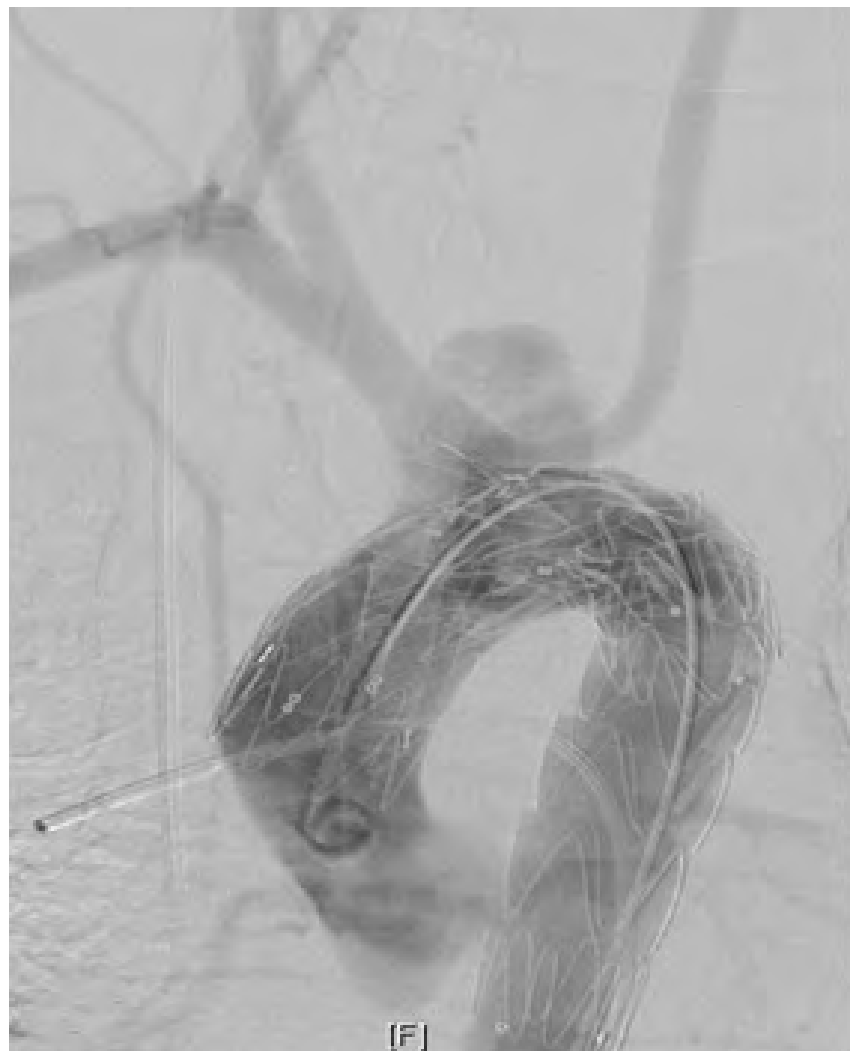

Figure 4. Post procedure visualization of Type $1 \mathrm{~A}$ endoleak with filling of the pseudoaneurysm.

between the endoprosthesis and the aortic wall, into the right common carotid artery in a U-shape pattern (see Figure 3 for a schematic version of the entire construction) [1]. The left subclavian artery received blood via a steal phenomenon through the left carotid artery system. The entire procedure was possible under low heparin dosage (Activated Clotting Time < 250 seconds) with immediate antagonistic Protamine administration afterwards.

Although the procedure went well, immediate control comparison revealed a type IA endoleak (Figure 4) which was treated additionally by direct suprasternal puncture of the pseudoaneurysm. Once the correct position of the sheath was verified, filling of the space with Gianturco coils (Cook Medical, Bloomington, Indiana, USA) and Glubran glue (GEM S.R.L., Lu, Italy) resulted in immediate success (Figure 5 ).

In the second stage, the femoral fracture was treated with external fixation. The patient was weaned from ventilation quite rapidly and further recovered

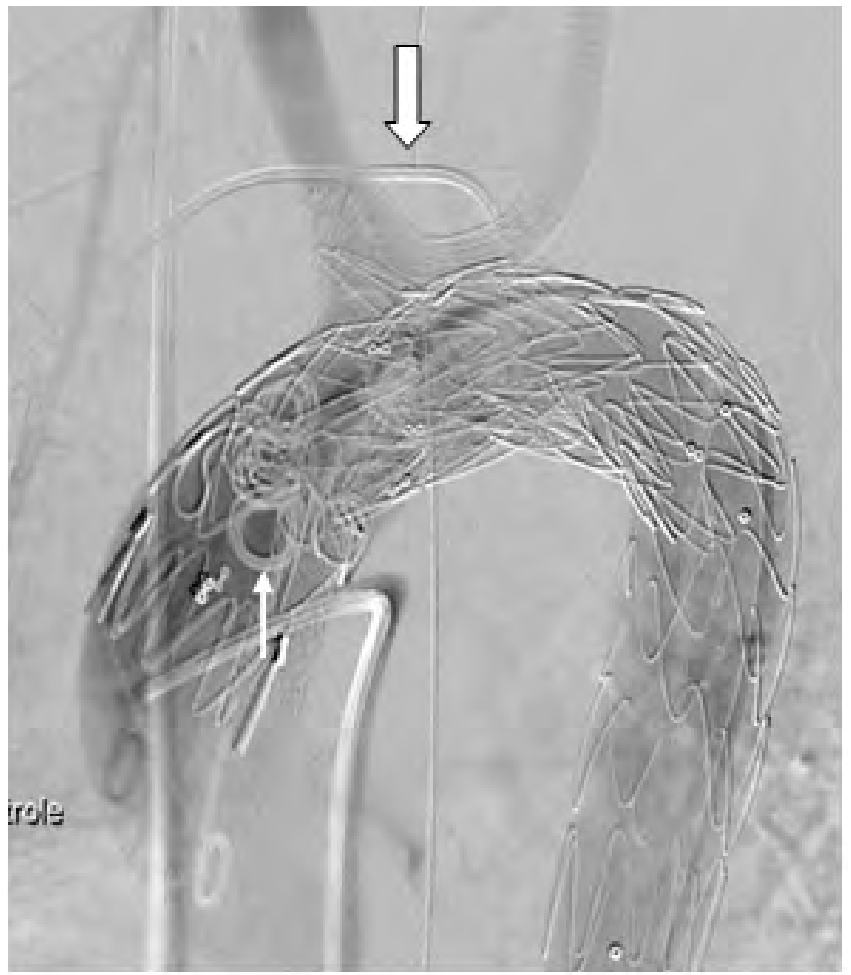

Figure 5. Post-treatment of the endoleak. Large white arrow: suprasternal sheath; Thin white arrow: coils.

without neurological sequelae. Finally, he was transferred for further rehabilitation to a specialized center.

Today, the patient is in perfect clinical condition without any problems regarding the extensive endovascular repair of his aortic arch and supraaortic vessels. A control CT scan and intraarterial angiogram 1 year later showed a good construction with patent chimneys and no type I or II leakage (Figure 6).

Follow up for this patient is life-long, as we expect future elective open reconstruction of the aortic arch due to his young age.

\section{Conflict of Interest}

The authors have no conflict of interest relevant to this publication.

Comment on this Article or Ask a Question 

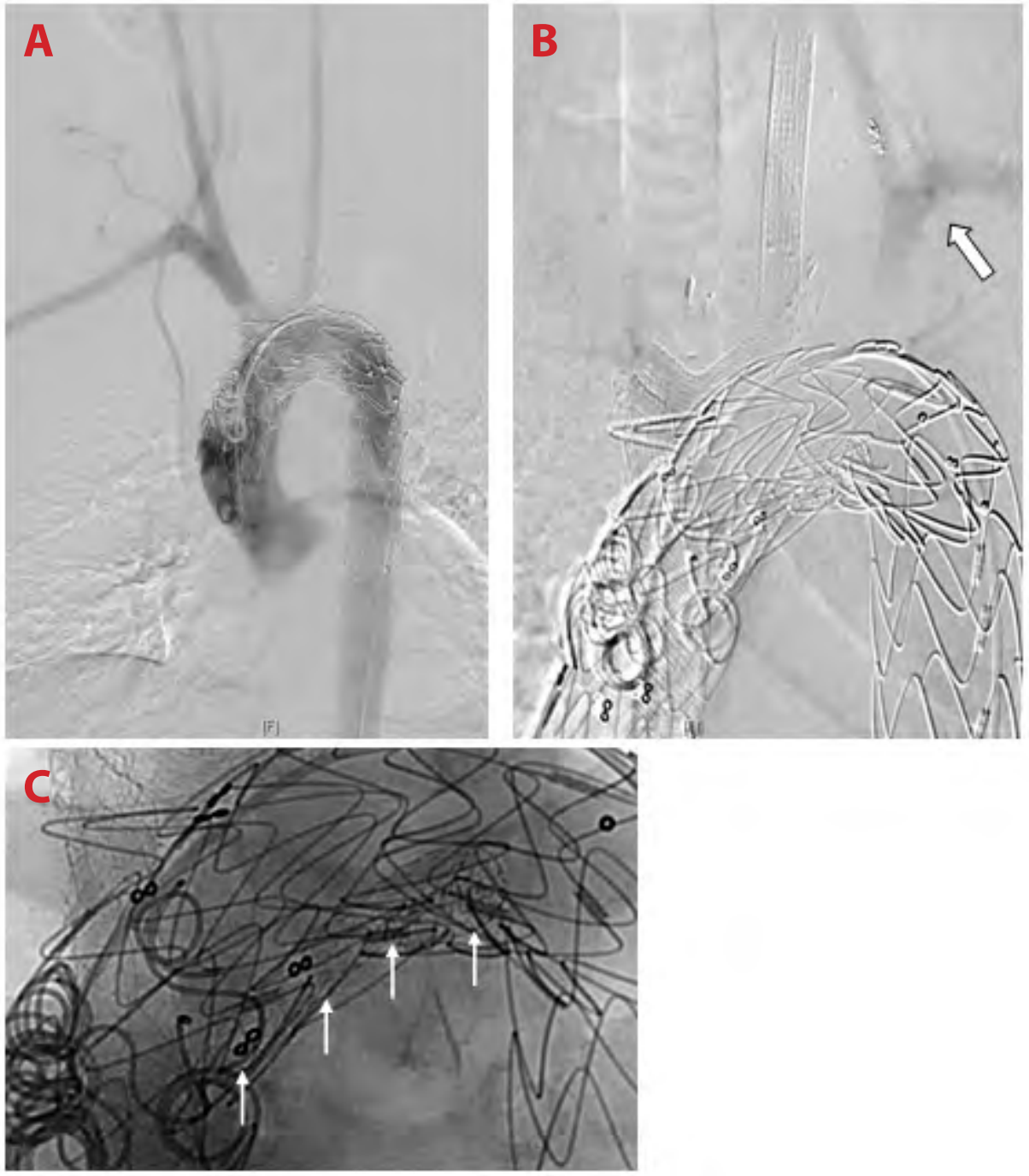

Figure 6. Stable control at 1 year. A) frontal view, early contrast phase. B) frontal view, late contrast phase. C) enhanced image. Note the subclavian steal to the left upper extremity (large arrow) and the luxated V12 stent still in situ (small white arrows).

\section{References}

1. Sakalihasan N, Limet $\mathrm{R}$, Defawe OD. Abdominal aortic aneurysm. Lancet. 2005;365:1577-1589. DOI: 10.1016/S01406736(05)66459-8.

2. Kuivaniemi $\mathrm{H}$, Tromp G, Carey DJ, Elmore JR. The molecular biology and genetics of aneurysms. In: Homeister JW and Willis MS. Molecular and Translational Vascular Medicine. New York: Springer Science+Business Media; 2012, p. 3-33. DOI: $\quad$ 10.1007/978-1-61779-906-8_1
Cite this article as: Hendriks $\mathrm{JMH}$, Brits T, Van der Zijden T, Monsieurs K, de Bock D, De Paep R. U-Shape Kissing Chimney TEVAR for a Traumatic Arch Rupture in a Polytraumatized Patient. AORTA. 2015;3(Issue 1): 41-45. DOI: http://dx.doi.org/10.12945/j.aorta.2015.14-044 


\section{EDITOR'S QUESTIONS}

1. You literally saved a life with your creativity. Do you have concerns about long-term durability of this endograft construct in an 18-year-old patient? Do you think the patient is susceptible to sudden brain events?

This indeed was an emergency solution for a young male polytraumatized patient who could not be helped by open cardiovascular surgery. As we stated at the end of the manuscript, intensive follow up is well organized, and elective surgery with reconstruction of the aortic arch has already been discussed with the patient. Because of his young age, we believe it is too hazardous to allow him to continue with a supraaortic blood supply that relies on a kissing stent solution.

For the moment, however, ultrasound and angiographic controls show good results with adequate flow and no signs of graft failure. For this reason, we don't expect short-term brain events and will thus allow the patient to focus on the last stages of his recovery. In the future, we will propose aortic arch replacement as a definitive long-term solution after informing the patient of the potential risks of follow-up therapy versus aortic arch surgery. 